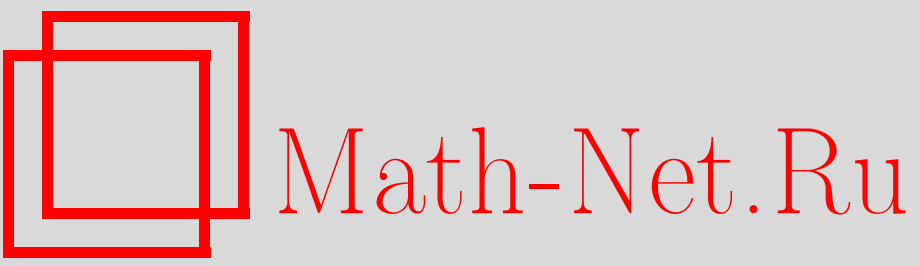

Е. Б. Яровая, Критерии экспоненциального роста числа частиц в моделях ветвящихся случайных блужданий, Теория вероятн. и ее примен., 2010, том 55, выпуск 4, 705-731

DOI: https://doi.org/10.4213/tvp4279

Использование Общероссийского математического портала Math-Net.Ru подразумевает, что вы прочитали и согласны с пользовательским соглашением

http: //www . mathnet.ru/rus/agreement

Параметры загрузки :

IP: 35.174 .16 .151

26 апреля 2023 г., 11:51:18 


\title{
КРИТЕРИИ ЭКСПОНЕНЦИАЛЬНОГО РОСТА ЧИСЛА ЧАСТИЦ В МОДЕЛЯХ ВЕТВЯЩИХСЯ СЛУЧАЙНЫХ БЛУЖДАНИЙ ${ }^{1)}$
}

\begin{abstract}
В статье рассматриваются две модели ветвящегося случайного блуждания с непрерывным временем на $\mathbf{Z}^{d}$ с одним источником ветвления. В первой из них случайное блуждание предполагается симметричным на всей решетке. Во второй модели с целью «искусственного» усиления степени преобладания свойства ветвления или блуждания в источнике вводится дополнительный параметр, что приводит к нарушению симметричности блуждания. Установлены необходимые и достаточные условия, при которых в моделях будет наблюдаться экспоненциальный рост численностей частиц как в произвольном узле, так и на всей решетке. Предложены общие методы исследования моделей в надкритическом случае.
\end{abstract}

Ключевые слова и фразы: ветвящиеся случайные блуждания, неоднородная среда, надкритический случай.

1. Введение. В последние годы ветвящиеся процессы с блужданием частиц получили название ветвящихся случайных блужданий или ветвящихся блужданий (ВСБ). Среди литературы, посвященной ВСБ, отметим одну из последних работ Б. А. Севастьянова [16].

Поведение ВСБ существенно зависит от структуры среды, в которой происходит блуждание частиц. При этом естественно возникает вопрос о влиянии неоднородности среды на предельное пространственное распределение частиц. Этот вопрос интересен также в связи с исследованием пространственных распределений в случайных средах с так называемыми «сильными центрами», в окрестности которых происходит основной рост процесса [7], [13]. Следует отметить также, что ВСБ с конечным числом источников ветвления могут быть использованы при описании широкого круга прикладных моделей, в частности, в теории массового обслуживания, а также в теории надежности [6], [25], [26]. Таким образом, анализ свойств ВСБ, связанных с неоднородностью, некомпактностью, а также размерностью пространства, может послужить для объяснения эффектов в более сложных неоднородных структурах.

\footnotetext{
*Московский государственный университет им. М.В.Ломоносова, Ленинские горы, 119991 Москва, ГСП-1, Россия; e-mail: yarovaya@mech.math.msu.su

1) Работа выполнена при поддержке РФФИ (грант № 10-01-00266).
} 
В статье рассматриваются две модели ВСБ с непрерывным временем на решетке $\mathbf{Z}^{d}$, в которых размножение и гибель частиц происходит в единственном узле решетки (источнике).

В обеих моделях исследуется эволюция системы частиц, состояние которой описывается числом частиц в момент времени $t$ в каждой точке $y \in \mathbf{Z}^{d}$, в предположении, что в начальный момент времени $t=0$ система состоит из одной частицы, находящейся в точке $x$. Частицы совершают случайное блуждание по точкам решетки $\mathbf{Z}^{d}$, причем в одной из точек решетки $x_{0}$ частицы могут к тому же размножаться и гибнуть. Без ограничения общности можно считать, что источник размножения и гибели частиц, $x_{0}$, расположен в начале координат. Предполагается также, что каждая из новых частиц эволюционирует по тому же закону, независимо от остальных частиц и от всей предыстории.

1.1. Модель I: симметричное ВСБ с одним источником. В этой модели случайное блуждание частиц полностью определяется матрицей переходных интенсивностей $A=(a(x, y))_{x, y \in \mathbf{Z}^{d}}$ и предполагается симметричным: $a(x, y)=a(y, x)$, однородным: $a(x, y)=a(0, y-x)=$ $a(y-x)$, неприводимым (т.е. любая точка $y \in \mathbf{Z}^{d}$ достижима), регулярным: $\sum_{x \in \mathbf{Z}^{d}} a(x)=0$, где $a(x) \geqslant 0$ при $x \neq 0$ и $a(0)<0$, с конечной дисперсией скачков: $\left.\sum_{x \in \mathbf{Z}^{d}}\|x\|^{2} a(x)<\infty\right)$, где $\|\cdot\|$ - евклидова норма вектора $x \in \mathbf{Z}^{d}$. В силу симметричности и однородности случайного блуждания $\sum_{y \in \mathbf{Z}^{d}} a(x, y)=\sum_{x \in \mathbf{Z}^{d}} a(x, y)=0$. Ветвление в источнике задается инфинитезимальной производящей функцией $f(u)=\sum_{n=0}^{\infty} b_{n} u^{n}$, где $b_{n} \geqslant 0$ при $n \neq 1, b_{1}<0$ и $\sum_{n} b_{n}=0$. Предполагается, что $\beta_{r}=f^{(r)}(1)<\infty$ при всех $r \in \mathbf{N}$. Величина $\beta_{1}=f^{\prime}(1)$ будет обозначаться через $\beta$.

Пусть $\mu_{t}(y)$ - число частиц в момент времени $t$ в точке $y$, тогда условие, что в начальный момент времени $t=0$ система состоит из одной частицы, находящейся в точке $x$, равносильно $\mu_{0}(y)=\delta_{y}(x)$. При этом общее число частиц на решетке определяется равенством $\mu_{t}=\sum_{y \in \mathbf{Z}^{d}} \mu_{t}(y)$. Таким образом, если в момент времени $t$ в точке $x_{0}=0$ находилось $\mu_{t}(0)>0$ частиц, то каждая из частиц, находящихся в точке $x_{0}=0$, может за малое время $h$ либо перейти с вероятностью $p(h ; 0, y)=a(y) h+o(h)$ в точку $y \neq 0$, либо произвести потомство из $n \neq 1$ частиц (мы считаем, что и сама частица входит в это число), либо погибнуть (случай, когда $n=0)$ с вероятностью $p_{*}(h ; n)=b_{n} h+o(h)$, либо сохраниться (никаких изменений не происходит) с вероятностью $1-\sum_{y \neq 0} a(y) h-\sum_{n \neq 1} b_{n} h+o(h)$. Отметим, что время пребывания частицы в источнике экспоненциально распределено с параметром $-\left(a(0)+b_{1}\right)$. В данной модели не предполагается независимость законов блуждания и ветвления в источнике, поскольку гибель и размножение происходят лишь при условии, что частица находится в источнике и не совершила скачка в какую-нибудь другую точку решетки за малое время $h$. 
Одно из первых исследований моделей такого типа проведено в работах [1], [3], [4], [21]-[24], в которых поведение средних численностей частиц как в произвольном узле, так и на всей решетке определяется структурой спектра линейного оператора $H=A+\beta \Delta_{0}$, где генератор случайного блуждания $A$ является ограниченным самосопряженным оператором в $l^{2}\left(\mathbf{Z}^{d}\right)$, а слагаемое $\beta \Delta_{0}$ «отвечает» за механизм ветвления в источнике (см. формальное определение $\Delta_{0}$ в п. 2.2 ).

1.2. Модель II: ВСБ с нарушением симметричности блуждания в источнике. При описании этой модели будем придерживаться терминологии, введенной в [14] для одномерной решетки. В модели II перемещение частиц по решетке $\mathbf{Z}^{d}$ (за исключением частиц, которые выходят из нуля) также происходит в соответствии с матрицей

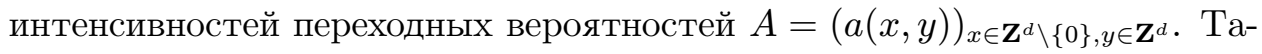
ким образом, поведение системы частиц вне источника в обеих моделях совпадает.

Интенсивности переходов из нуля в модели II пропорциональны интенсивностям переходов из других состояний и имеют вид $a(0, y)=$ $-a(y) / a(0), y \neq 0$. Это означает, что вероятности переходов из одного узла решетки в другой для блуждания однородны по пространству и времени, причем время пребывания в источнике имеет экспоненциальное распределение с параметром 1 , тогда как время пребывания в любом другом состоянии экспоненциально распределено с параметром $-a(0)$.

Развитие популяции начинается в момент времени $t=0 \mathrm{c}$ одной частицы, расположенной в произвольном узле $x$ (в [5], [6], [10], [14] рассмотрен случай старта процесса из нуля). Начальная частица, так же как и любая другая, оказавшаяся в нуле позднее, проводит в этой точке случайное время, имеющее экспоненциальное распределение с единичным средним, а затем либо переходит в точку $y \neq 0$ с вероятностью $(1-\alpha) a(0, y)$, либо гибнет с вероятностью $\alpha$, порождая при этом случайное количество потомков $\zeta$ в соответствии с производящей функцией $\overline{\mathscr{F}}(u)=\mathbf{E} u^{\zeta}=\sum_{n=0}^{\infty} f_{n} u^{n}$ (здесь и ниже черта над функцией обозначает, что рассматриваемая функция относится к модели II; отметим, что в [5], [6] функция $\overline{\mathscr{F}}(u)$ обозначена через $f(u))$. Предполагается, что математическое ожидание $\mathbf{E} \zeta$ и дисперсия $\mathbf{D} \zeta^{2}$ случайной величины $\zeta$ конечны, а дисперсия, кроме того, отлична от нуля: $\mathbf{E} \zeta<\infty$, $\sigma^{2}=\mathbf{D} \zeta^{2} \in(0, \infty)$

Так же как и в модели I, основными объектами исследования в модели II является число частиц $\bar{\mu}_{t}(y)$ в момент времени $t$ в точке $y$ и общее число частиц на решетке $\bar{\mu}_{t}=\sum_{y} \bar{\mu}_{t}(y)$. Тогда условие, что в начальный момент система состоит из одной частицы, находящейся в точке $x$, равносильно $\bar{\mu}_{0}(y)=\delta_{y}(x)$.

Отличительной особенностью этой модели является введение параметра $\alpha$, управляющего поведением процесса в источнике ветвления и, в 
результате, приводящего к нарушению симметричности матрицы переходных интенсивностей $A$. При $\mathbf{E} \zeta=1$ и $d=1,2$ данная модель изучена в [5], [6], [10], [14], где основной идеей исследования явилось представление ВСБ как ветвящегося процесса Беллмана-Харриса с двумя типами частиц. В [5], [6], [14] такие ВСБ названы каталитическими.

Удобным инструментом описания процесса ветвления с непрерывным временем является инфинитезимальная производящая функция [27]

$$
\bar{f}(u)=\alpha\left(\sum_{n \neq 1}^{\infty} f_{n} u^{n}+\left(f_{1}-1\right) u\right)
$$

Предположим, как и в модели I, что $\bar{\beta}_{r}=\bar{f}^{(r)}(1)<\infty$ при всех $r$, т.е. число потомков частицы имеет конечные моменты всех порядков. Будем обозначать далее $\bar{\beta}=\bar{\beta}_{1}=\bar{f}^{\prime}(1)$.

1.3. Структура работы и краткое изложение основных результатов. Несмотря на близкое описание эволюции частиц в обеих моделях и получающиеся сходные результаты, сравнение этих моделей вызывало определенные затруднения. В связи с этим в [27] было предложено единое описание изучаемых ВСБ в терминах «инфинитезимальных» характеристик и на его основе получены однотипные эволюционные уравнения. Такой подход дает возможность применить для исследования ВСБ с нарушением симметричности блуждания в источнике те же методы описания процесса в виде бесконечномерных дифференциальных уравнений, которые были развиты ранее для исследования симметричного ВСБ.

Применение этих уравнений позволяет получить один из основных результатов разд. 2, согласно которому эволюция средних численностей частиц во второй модели определяется структурой спектра оператора $\bar{H}=\bar{A}+\bar{\beta} \Delta_{0}$, где генератор случайного блуждания $\bar{A}$ является ограниченным (но уже, вообще говоря, не симметричным, как в первой модели) оператором в $l^{2}\left(\mathbf{Z}^{d}\right)$, а слагаемое $\bar{\beta} \Delta_{0}$, как и в первой модели, характеризует механизм ветвления в источнике.

В [1], [3], [23] показано, что если в спектре оператора $H$ содержится собственное значение $\lambda>0$, то в модели I при $t \rightarrow \infty$ происходит экспоненциальный рост как локальных численностей частиц, так и их полного числа:

$$
\lim _{t \rightarrow \infty} \mu_{t}(y) e^{-\lambda t}=\varphi \psi_{\lambda}(y), \quad \lim _{t \rightarrow \infty} \mu_{t} e^{-\lambda t}=\varphi
$$

где $\psi_{\lambda}(y)$ - некоторая функция, а $\varphi$ - невырожденная случайная величина. При доказательстве этих утверждений существенна симметричность ВСБ, которая и определяет самосопряженность оператора $H$.

В разделе 3 удается показать, что спектральные свойства оператора $\bar{H}$, несмотря на отсутствие у него свойства самосопряженности, во 
многом аналогичны спектральным свойствам оператора $H$. В частности, в теоремах 3.1 и 3.2 устанавливаются необходимые и достаточные условия существования и единственности у оператора $\bar{H}$ изолированного положительного собственного значения.

Проведенный в разд. 3 спектральный анализ оператора $\bar{H}$ позволяет получить в разделе 4 для второй модели соотношения, справедливые в смысле сходимости моментов, аналогичные (1):

$$
\lim _{t \rightarrow \infty} \bar{\mu}_{t}(y) e^{-\bar{\lambda} t}=\xi \psi_{\bar{\lambda}}(y), \quad \lim _{t \rightarrow \infty} \bar{\mu}_{t} e^{-\bar{\lambda} t}=\xi,
$$

где $\psi_{\bar{\lambda}}(y)$ - некоторая функция, $\xi-$ невырожденная случайная величина, а $\bar{\lambda}$ - единственное изолированное положительное собственное значение оператора $\bar{H}$. Здесь же приведены условия, при которых моменты однозначно определяют распределение случайной величины $\xi$ и, таким образом, соотношения (2) справедливы в смысле сходимости по распределению.

Важным следствием теорем 3.1 и 3.2 является тот факт, что во второй модели, так же как и в первой [23], для каждой размерности решетки при изменении параметра $\bar{\beta}$ возможны три основных типа предельного поведения средних численностей частиц как в произвольном узле, так и на всей решетке, а именно, существует некоторое значение $\bar{\beta}_{\mathrm{c}}$ такое, что при $\bar{\beta}<\bar{\beta}_{\mathrm{c}}, \bar{\beta}=\bar{\beta}_{\mathrm{c}}$ и $\bar{\beta}>\bar{\beta}_{\mathrm{c}}$ решения эволюционных уравнений $\partial_{t} m_{1}=\bar{H} m_{1}$ различны. В этом смысле $\bar{\beta}_{\text {с }}$ естественно назвать критическим значением ВСБ.

В заключительном разд. 5 предлагается схема исследования ВСБ с конечным числом источников ветвления, обобщающая подход, развитый в разд. $2-4$.

2. Основные уравнения. В этом разделе выписаны уравнения для переходных вероятностей случайного блуждания, для производящих функций числа частиц в произвольном узле $y \in \mathbf{Z}^{d}$ и общей численности частиц в моделях I и II. Установлены соотношения между переходными вероятностями случайного блуждания (без ветвления) и их преобразованиями Лапласа в этих моделях ВСБ. Получены рекуррентные уравнения для моментов в модели II.

2.1. Уравнения для переходных вероятностей и производящих функций. Из описания модели I вытекают соотношения для переходной вероятности случайного блуждания $p(h ; x, y):$ при малых $h$

$$
\begin{aligned}
& p(h ; x, y)=a(x, y) h+o(h) \quad \text { при } \quad y \neq x, \\
& p(h ; x, x)=1+a(x, x) h+o(h) .
\end{aligned}
$$

Тогда (см., например, [8]) $p(t ; x, y)$ удовлетворяет системе обратных уравнений Колмогорова

$$
\partial_{t} p(t ; x, y)=A p(t ; x, y), \quad p(0 ; x, y)=\delta_{y}(x),
$$


где $\delta_{y}(\cdot)$ - дискретная $\delta$-функция Кронекера на $\mathbf{Z}^{d}$, а $A p(t ; x, y)=$ $\sum_{x^{\prime}} a\left(x, x^{\prime}\right) p\left(t ; x^{\prime}, y\right)$.

Аналогично устанавливается, что переходные вероятности $\bar{p}(t ; x, y)$ в модели II удовлетворяют системе дифференциальных уравнений

$$
\partial_{t} \bar{p}(t ; x, y)=\bar{A} \bar{p}(t ; x, y), \quad \bar{p}(0 ; x, y)=\delta_{y}(x),
$$

где $\bar{A} \bar{p}(t ; x, y)=\sum_{x^{\prime}} \bar{a}\left(x, x^{\prime}\right) \bar{p}\left(t ; x^{\prime}, y\right)$ и при этом $\bar{a}\left(x, x^{\prime}\right)=\left(1-\delta_{0}(x)(1-\right.$ $(\alpha-1) / a(0))) a\left(x, x^{\prime}\right)$.

Определим производящие функции для случайных величин $\bar{\mu}_{t}(y)$ и $\bar{\mu}_{t}$ при $z \geqslant 0$ в модели II равенствами $\bar{F}(z, t ; x, y)=\mathbf{E}_{x} e^{-z \bar{\mu}_{t}(y)}$ и $\bar{F}(z, t ; x)=\mathbf{E}_{x} e^{-z \bar{\mu}_{t}}$. Тогда, как показано в [27], при каждом $0 \leqslant z \leqslant \infty$ функции $\bar{F}(z, t ; x)$ и $\bar{F}(z, t ; x, y)$ непрерывно дифференцируемы по переменной $t$ равномерно по $x, y \in \mathbf{Z}^{d}$, удовлетворяют неравенствам $0 \leqslant$ $\bar{F}(z, t ; x), \bar{F}(z, t ; x, y) \leqslant 1$ и дифференциальным уравнениям

$$
\begin{aligned}
\partial_{t} \bar{F}(z, t ; x) & =(\bar{A} \bar{F}(z, t ; \cdot))(x)+\delta_{0}(x) \bar{f}(\bar{F}(z, t ; x)), \\
\partial_{t} \bar{F}(z, t ; x, y) & =(\bar{A} \bar{F}(z, t ; \cdot, y))(x)+\delta_{0}(x) \bar{f}(\bar{F}(z, t ; x, y))
\end{aligned}
$$

с начальными условиями $\bar{F}(z, 0 ; x)=e^{-z}$ и $\bar{F}(z, 0 ; x, y)=e^{-z \delta_{y}(x)}$ соответственно. При этом выражение $\bar{A} \bar{F}(z, t ; \cdot)$ следует понимать как единый символ, обозначающий зависящую от параметра $z$ и времени $t$ функцию $(\bar{A} \bar{F}(z, t ; \cdot))(x)=\sum_{x^{\prime} \in \mathbf{Z}^{d}} \bar{a}\left(x, x^{\prime}\right) \bar{F}\left(z, t ; x^{\prime}\right)$ переменной $x \in \mathbf{Z}^{d}$. Аналогично определяется и выражение $\bar{A} \bar{F}(z, t ; \cdot, y)$ в формуле (4).

Как показано в [2], [23], [24], уравнения (3) и (4) также описывают и поведение производящих функций $F(z, t ; x, y)=\mathbf{E}_{x} e^{-z \mu_{t}(y)}$ и $F(z, t ; x)=$ $\mathbf{E}_{x} e^{-z \mu_{t}}$ в модели I, если заменить в них символы «с черточками» на соответствующие символы «без черточек».

Сравним уравнения (3) и (4) для моделей I и II. Сходство заключается в том, что операторы $A$ и $\bar{A}$ являются линейными ограниченными операторами, действующими в $l^{q}\left(\mathbf{Z}^{d}\right)$. Однако в силу симметрии случайного блуждания оператор $A$ является самосопряженным, а оператор $\bar{A}$ этим свойством не обладает.

2.2. Связь между переходными вероятностями в моделях. Вначале рассмотрим в пространстве $l^{2}\left(\mathbf{Z}^{d}\right)$ однородное дифференциальное уравнение с оператором $A$

$$
\frac{d u}{d t}=A u
$$

Решение задачи Коши для этого уравнения с начальным условием $u(0)=\delta_{0}$ может быть представлено в терминах оператор-функции $e^{A t}$ в виде $u(t)=e^{A t} \delta_{0}$. Если $\delta_{0}=\delta_{0}(\cdot)$, как обычно, обозначает вектор-столбец на решетке, принимающий единичное значение в начале координат, и 
нули в остальных точках, то $\Delta_{0}$ определим равенством $\Delta_{0}=\delta_{0} \delta_{0}^{T}$. Положим $a=a(0)$ и $s=1-a(\alpha-1)^{-1}$, тогда $\bar{A}=A-\Delta_{0} A+(\alpha-1) a^{-1} \Delta_{0} A=$ $A+(\alpha-1) a^{-1} s \Delta_{0} A=A+(1-s)^{-1} s \Delta_{0} A$. Поэтому с помощью метода вариации произвольной постоянной решение задачи Коши для уравнения

$$
\frac{d \bar{u}}{d t}=\bar{A} \bar{u} \equiv A \bar{u}+(1-s)^{-1} s \Delta_{0} A \bar{u}
$$

с начальным условием $\bar{u}(0)=\delta_{0}$ может быть представлено в виде

$$
\bar{u}(t)=e^{A t} \delta_{0}+\int_{0}^{t} e^{A(t-v)}(1-s)^{-1} s \Delta_{0} A \bar{u}(v) d v
$$

или, что то же в силу соотношения $\Delta_{0}=\delta_{0} \delta_{0}^{T}$,

$$
\bar{u}(t)=e^{A t} \delta_{0}+(1-s)^{-1} s \int_{0}^{t} e^{A(t-v)} \delta_{0} \delta_{0}^{T} A \bar{u}(v) d v .
$$

Здесь $\delta_{0}^{T} A \bar{u}(t) \equiv\left\langle A \bar{u}(t), \delta_{0}\right\rangle$ - числовая функция, где $\langle\cdot, \cdot\rangle$ обозначает евклидово скалярное произведение в $l^{2}\left(\mathbf{Z}^{d}\right)$. Тогда в силу соотношения $u(t)=e^{A t} \delta_{0}$ имеем $e^{A(t-v)} \delta_{0}=u(t-v)$, и поэтому

$$
\bar{u}(t)=u(t)+(1-s)^{-1} s \int_{0}^{t}\left(e^{A(t-v)} \delta_{0}\right)\left\langle A \bar{u}(v), \delta_{0}\right\rangle d v .
$$

Отсюда, полагая $u(t ; \cdot)=p(t ; \cdot, y), \bar{u}(t ; \cdot)=\bar{p}(t ; \cdot, y)$ и учитывая, что $e^{A(t-v)} \delta_{0}=p(t-v ; \cdot, 0)$, получаем

$$
\bar{p}(t ; x, y)=p(t ; x, y)+(1-s)^{-1} s \int_{0}^{t} p(t-v ; x, 0)\left(\sum_{x^{\prime} \in \mathbf{Z}^{d}} a\left(x^{\prime}\right) \bar{p}\left(v ; x^{\prime}, y\right)\right) d v
$$

Выражение в скобках под знаком интеграла есть $A \bar{p}(v ; 0, y)$. А так как в силу (5) имеет место соотношение $A \bar{p}(t ; 0, y)=(1-s) \partial_{t} \bar{p}(t ; 0, y)$, то (6) влечет равенство

$$
\bar{p}(t ; x, y)=p(t ; x, y)+s \int_{0}^{t} p(t-v ; x, 0) \partial_{v} \bar{p}(v ; 0, y) d v .
$$

Обозначим для краткости $p(t)=p(t ; 0,0), \bar{p}(t)=\bar{p}(t ; 0,0)$. Тогда уравнение (7) при $x=y=0$ примет вид

$$
\bar{p}(t)=p(t)+s \int_{0}^{t} p(t-v) \partial_{v} \bar{p}(v) d v .
$$

При $\lambda \geqslant 0$ положим

$$
\begin{array}{ll}
G_{\lambda}(x, y)=\int_{0}^{\infty} e^{-\lambda t} p(t ; x, y) d t, & G_{\lambda}=\int_{0}^{\infty} e^{-\lambda t} p(t) d t \\
\bar{G}_{\lambda}(x, y)=\int_{0}^{\infty} e^{-\lambda t} \bar{p}(t ; x, y) d t, & \bar{G}_{\lambda}=\int_{0}^{\infty} e^{-\lambda t} \bar{p}(t) d t .
\end{array}
$$


Сделаем несколько замечаний о свойствах функций $G_{\lambda}(x, y)$ и $\bar{G}_{\lambda}(x, y)$. Сначала заметим, что по вероятностному смыслу функции $p(t ; x, y)$ при всех $x, y \in \mathbf{Z}^{d}$ и $t \geqslant 0$ справедливы неравенства $\sum_{x^{\prime}} p\left(t ; x^{\prime}, y\right) \leqslant 1$ и $\sum_{y^{\prime}} p\left(t ; x, y^{\prime}\right) \leqslant 1$. В настоящий момент не важно, что данные неравенства на самом деле превращаются в равенства при сделанных выше предположениях о неприводимости случайного блуждания. Тогда в силу (8) функция $G_{\lambda}(x, y)$ при $\lambda>0$ также оказывается суммируемой по каждой из переменных $x, y \in \mathbf{Z}^{d}$ :

$$
\begin{aligned}
\sum_{x^{\prime}} G_{\lambda}\left(x^{\prime}, y\right) & =\sum_{x^{\prime}} \int_{0}^{\infty} e^{-\lambda t} p(t ; x, y) d t \\
& =\int_{0}^{\infty} e^{-\lambda t} \sum_{x^{\prime}} p\left(t ; x^{\prime}, y\right) d t \leqslant \int_{0}^{\infty} e^{-\lambda t} d t \leqslant \frac{1}{\lambda}
\end{aligned}
$$

Аналогичные соотношения верны и при суммировании функции $G_{\lambda}(x, y)$ по второму аргументу, а также при суммировании по каждому из аргументов функции $\bar{G}_{\lambda}(x, y)$. Поэтому

$$
\begin{aligned}
& \sum_{x^{\prime}} G_{\lambda}\left(x^{\prime}, y\right) \leqslant \frac{1}{\lambda}, \quad \sum_{y^{\prime}} G_{\lambda}\left(x, y^{\prime}\right) \leqslant \frac{1}{\lambda}, \\
& \sum_{x^{\prime}} \bar{G}_{\lambda}\left(x^{\prime}, y\right) \leqslant \frac{1}{\lambda}, \quad \sum_{y^{\prime}} \bar{G}_{\lambda}\left(x, y^{\prime}\right) \leqslant \frac{1}{\lambda}
\end{aligned}
$$

при $\lambda>0$ и всех $x, y \in \mathbf{Z}^{d}$.

В силу равенства $\int_{0}^{\infty} e^{-\lambda t} \partial_{t} \bar{p}(t) d t=\lambda \bar{G}_{\lambda}-1$ после применения преобразования Лапласа к каждой части уравнения (7) получаем

$$
\bar{G}_{\lambda}(x, 0)=G_{\lambda}(x, 0)+s G_{\lambda}(x, 0)\left(\lambda \bar{G}_{\lambda}-1\right)
$$

откуда при $x=0$ вытекает соотношение

$$
\bar{G}_{\lambda}=(1-s) G_{\lambda}\left(1-\lambda s G_{\lambda}\right)^{-1}
$$

В дальнейшем нам также понадобится результат о предельном поведении переходных вероятностей $p(t ; x, y)$ в модели I, которые имеют степенную асимптотику по $t$ (см., например, [23]), причем первый член асимптотического разложения $p(t ; x, y)$ не зависит от $x$ и $y$ :

$$
p(t ; x, y) \sim \gamma_{d} t^{-d / 2} \quad \text { при } t \rightarrow \infty,
$$

где $\gamma_{d}>0$ - некоторая константа. 
2.3. Дифференциальные уравнения для моментов. Классификация асимптотического поведения моментов случайных величин $\mu_{t}$ и $\mu_{t}(y)$ для модели I проведена в [23]. Сформулируем ряд аналогичных утверждений для модели II. Из свойств преобразования Лапласа [18] следует, что производящие функции $\bar{F}(z, t ; x)=\mathbf{E}_{x} e^{-z \bar{\mu}_{t}}$ и $\bar{F}(z, t ; x, y)=$ $\mathbf{E}_{x} e^{-z \bar{\mu}_{t}(y)}$ бесконечно дифференцируемы по $z$ при $z>0$ и их производные имеют вид

$$
\begin{aligned}
\partial_{z}^{n} \bar{F}(z, t ; x) & =(-1)^{n} \mathbf{E}_{x} \bar{\mu}_{t}^{n} e^{-z \bar{\mu}_{t}}, \\
\partial_{z}^{n} \bar{F}(z, t ; x, y) & =(-1)^{n} \mathbf{E}_{x} \bar{\mu}_{t}^{n}(y) e^{-z \bar{\mu}_{t}(y)} .
\end{aligned}
$$

Положив в предыдущих соотношениях $z=0$, получаем представления для моментов:

$$
\begin{aligned}
m_{n}(t ; x) & =\mathbf{E}_{x} \bar{\mu}_{t}^{n}=\left.(-1)^{n} \partial_{z}^{n} \bar{F}(z, t ; x)\right|_{z=0}, \\
m_{n}(t ; x, y) & =\mathbf{E}_{x} \bar{\mu}_{t}^{n}(y)=\left.(-1)^{n} \partial_{z}^{n} \bar{F}(z, t ; x, y)\right|_{z=0} .
\end{aligned}
$$

Определим операторы $H=A+\beta \Delta_{0}$ и $\bar{H}=\bar{A}+\bar{\beta} \Delta_{0}=(I+(1-$ $\left.s)^{-1} s \Delta_{0}\right) A+\bar{\beta} \Delta_{0}$. Оператор $H$ действует в каждом из пространств $l^{q}\left(\mathbf{Z}^{d}\right), 1 \leqslant q \leqslant \infty[23]$. Аналогичное утверждение справедливо и для оператора $\bar{H}$, и, более того, для модели II справедлив следующий аналог теоремы 1.3.1 из [23].

Теорема 2.1. При каждом иелом $k \geqslant 1$ моменть $m_{k}(t ; x, y) u$ $m_{k}(t ; x)$ в модели II удовлетворяют дифференииальньц уравнениям

$$
\begin{aligned}
\partial_{t} m_{1} & =\bar{H} m_{1}, \\
\partial_{t} m_{k} & =\bar{H} m_{k}+\delta_{0}(x) g_{k}\left(m_{1}, \ldots, m_{k-1}\right), \quad k \geqslant 2,
\end{aligned}
$$

с начальными условиями $m_{n}(0 ; \cdot, y)=\delta_{y}(\cdot)$ и $m_{n}(0 ; \cdot) \equiv 1$ соответственно. Здесь $\bar{H} m_{k}$ есть сокращенная запись для $\left(\bar{H} m_{k}(t ; \cdot, y)\right)(x)$ или $\left(\bar{H} m_{k}(t ; \cdot)\right)(x)$ соответственно и

$$
g_{k}\left(m_{1}, \ldots, m_{k-1}\right)=\sum_{r=2}^{k} \frac{\bar{\beta}_{r}}{r !} \sum_{\substack{i_{1}, \ldots, i_{r}>0 \\ i_{1}+\cdots+i_{r}=k}} \frac{k !}{i_{1} ! \cdots i_{r} !} m_{i_{1}} \cdots m_{i_{r}} .
$$

В разделе 4 показывается, что теорема 2.1 влечет для модели II соотношения типа (1). Доказательство будет опираться на анализ спектральных свойств оператора $\bar{H}$.

3. Спектральные свойства оператора $\bar{H}$. Как было установлено в [1], [3], [4], [21], [23], для экспоненциального роста численностей частиц в модели I необходимо и достаточно, чтобы выполнялось условие

$$
\beta>\beta_{\mathrm{c}}=G_{0}^{-1} .
$$


При этом значение $\beta_{\mathrm{c}}=G_{0}^{-1}$ параметра $\beta_{\mathrm{c}}$ было названо критическим. В этом разделе показывается, что в модели II аналогом условия (16) является неравенство

$$
\bar{\beta}>\bar{G}_{0}^{-1} .
$$

Напомним, что по предположению $a=a(0)<0$ и $0<\alpha<1$, и поэтому всюду ниже $s=1-a(\alpha-1)^{-1}<1$.

Теорема 3.1. Спектр оператора $\bar{H}$ расположен на вещественной оси, причем все положительные точки спектра оператора $\bar{H}$, если таковые существуют, являются собственньли значениями. При этом справедливы следующие утверждения:

а) число $\lambda>0$ является собственным значением оператора $\bar{H}$ тогда и только тогда, когда оно является корнем уравнения

$$
\bar{\beta} \bar{G}_{\lambda}=1 ;
$$

б) собственное значение $\lambda>0$ оператора $\bar{H}$ простое, и собственный вектор $g$, отвечаюший $\lambda$, определяется равенством

$$
g(x)=g(0) \bar{G}_{\lambda}^{-1} \bar{G}_{\lambda}(x, 0), \quad g(0) \neq 0, \quad x \in \mathbf{Z}^{d} ;
$$

в) спектральный проектор $\bar{P}$ оператора $\bar{H}$ на одномерное собственное подпространство, отвечающее собственному значению $\lambda>0$, имеет вид

$$
\bar{P} u=\frac{\langle g, u\rangle-s\left\langle\delta_{0}, g\right\rangle\left\langle\delta_{0}, u\right\rangle}{\langle g, g\rangle-s\left\langle\delta_{0}, g\right\rangle^{2}} g \quad \forall u \in l^{2}\left\langle\mathbf{Z}^{d}\right),
$$

где $\langle\cdot, \cdot\rangle-$ евклидово скалярное произведение в $l^{2}\left(\mathbf{Z}^{d}\right)$.

Теорема 3.2. Пусть $s<1$, тогда справедливы следующие утверждения:

а) оператор $\bar{H}$ обладает хотя бы одним положительным собственным значением тогда и только тогда, когда выполнено условие (17);

б) при выполнении условия (17) оператор $\bar{H}$ имеет единственное собственное значение.

3.1. Доказательство теоремы 3.1. Далее нам потребуются леммы 3.1-3.3, доказательства которых для удобства изложения вынесены в п. 3.3 .

Лемма 3.1. Пусть $s<1$. Тогда оператор

$$
D=I+\left(\sqrt{1+(1-s)^{-1} s}-1\right) \Delta_{0}
$$

обратим, а оператор $D^{-1} \bar{H} D$ имеет вид $D^{-1} \bar{H} D=D A D+\bar{\beta} \Delta_{0}$ и является самосопряженным. 
По лемме 3.1 оператор $\bar{H}$ подобен самосопряженному оператору, и значит, его спектр лежит на вещественной оси. Для доказательства того факта, что оператор $\bar{H}$ может иметь в положительной части вещественной оси только изолированные собственные значения, заметим, что в силу равенства $\bar{H}=A+(1-s)^{-1} s \Delta_{0} A+\bar{\beta} \Delta_{0}$ оператор $\bar{H}$ является конечномерным возмущением (оператор $\Delta_{0}=\delta_{0} \delta_{0}^{T}$ действует на одномерное пространство, натянутое на вектор $\delta_{0}$ ) самосопряженного оператора $A$. Тогда по теореме Вейля [11] существенные спектры операторов $A$ и $\bar{H}$ совпадают, а вне множества, совпадающего с существенным спектром оператора $A$, оператор $\bar{H}$ может иметь лишь изолированные собственные значения. Поскольку существенный спектр оператора $A$ (будучи частью всего его спектра) лежит в отрицательной части вещественной оси, то в положительной части вещественной оси оператор $\bar{H}$ может иметь только изолированные собственные значения, что и доказывает первую часть теоремы.

Утверждения а) и б) теоремы 3.1 вытекают из приводимой ниже леммы.

Лемма 3.2. Пусть $s<1$. Число $\lambda>0$ является собственнылм значением оператора $\bar{H}$ тогда и только тогда, когда оно удовлетворяет уравнению (18). При этом собственньй вектор $g$, отвечающий $\lambda>0$, определяется равенством (19) и каждое собственное значение $\lambda>0$ onepamopa $\bar{H}$ nростое.

Наконец, утверждение в) теоремы 3.1 вытекает из следующей леммы.

Лемма 3.3. Спектральный проектор оператора $\bar{H}$ на одномерное собственное подпространство, отвечающее простому собственному значению $\lambda>0$, имеет вид (20).

Доказательство теоремы 3.1 завершено.

3.2. Доказательство теоремы 3.2. Для доказательства утверждения а) установим сначала, что условие $\bar{\beta}>\bar{G}_{0}^{-1}$ влечет существование у оператора $\bar{H}$ хотя бы одного положительного собственного значения. Положив $\lambda=0$ в (11), получаем равенство $\bar{G}_{0}=(1-s) G_{0}$, в силу которого условие (17) может быть записано в виде

$$
\bar{\beta}>(1-s)^{-1} \beta_{\mathrm{c}} .
$$

Достаточность условия (22) для существования у оператора $\bar{H}$ хотя бы одного положительного собственного значения доказана в работе [27], тем не менее, для полноты изложения повторим соответствующие рассуждения ниже.

В силу утверждения а) теоремы 3.1 задача о существовании собственного значения $\lambda>0$ оператора $\bar{H}$ сводится к нахождению положи- 
тельного решения $\lambda$ уравнения (18). С помощью равенства (11) представим уравнение (18) в виде

$$
((1-s) \bar{\beta}+s \lambda)^{-1}=G_{\lambda} .
$$

С учетом (8) и (12) функция $G_{\lambda}$ положительна и строго монотонно убывает по $\lambda$, причем $\lim _{\lambda \rightarrow \infty} G_{\lambda}=0$; если же $\lambda \rightarrow 0$, то функция $G_{\lambda}$ неограниченно возрастает при $d=1,2$ и $\lim _{\lambda \rightarrow 0} G_{\lambda}=\beta_{\text {с }}^{-1}$ при $d \geqslant 3$.

По условию $\bar{\beta}>(1-s)^{-1} \beta_{\text {c }}$. Тогда для $s \leqslant 0$ левая часть уравнения (23) на интервале $\lambda \in\left(0,(s-1) s^{-1} \bar{\beta}\right)$ положительна и строго монотонно возрастает по $\lambda$, а при $\lambda>(s-1) s^{-1} \bar{\beta}$ отрицательна. Поэтому при $s \leqslant 0$ у уравнения (23) существует положительное решение, причем это решение единственно и принадлежит интервалу $\left(0,(s-1) s^{-1} \bar{\beta}\right)$.

Теперь рассмотрим оставшийся случай $0<s<1$. В этом предположении левая часть уравнения (23) положительна и строго убывает при всех $\lambda>0$. Более того,

$$
G_{\lambda}>((1-s) \bar{\beta}+s \lambda)^{-1}
$$

в сколь угодно малой окрестности нуля. При $d \geqslant 3$ неравенство (24) следует из того факта, что при $\lambda=0$ это неравенство принимает вид $G_{0}>((1-s) \bar{\beta})^{-1}$, что равносильно в силу (11) неравенству $\bar{G}_{0}>\bar{\beta}^{-1}$, выполняющемуся по условию (17). При $d=1,2$ неравенство (24) также верно, поскольку в этом случае $G_{\lambda}$ неограниченно возрастает, а левая часть уравнения (23) ограничена в малой окрестности нуля.

Для завершения доказательства достаточно показать, что

$$
G_{\lambda}<((1-s) \bar{\beta}+s \lambda)^{-1}
$$

при некоторых $\lambda>0$. Для этого представим функцию $G_{\lambda}$ в виде

$$
G_{\lambda}=\int_{0}^{\infty} e^{-\lambda t} p(t) d t=\lambda^{-1}+\lambda^{-1} \int_{0}^{\infty} e^{-\lambda t} p^{\prime}(t) d t
$$

Здесь $p(t)$ в силу [6], [23] не возрастает, а ее производная $p^{\prime}(t)<0$ не убывает и, значит,

$$
G_{\lambda}=\lambda^{-1}+r(\lambda), \quad r(\lambda)=\lambda^{-1} \int_{0}^{\infty} e^{\lambda t} p^{\prime}(t) d t<0,
$$

откуда следует, что (25) будет выполнено при таких значениях $\lambda$, для которых $\lambda^{-1}<((1-s) \bar{\beta}+s \lambda)^{-1}$. Но последнее неравенство выполняется при любых $\lambda>\bar{\beta}$. Таким образом, положительное решение уравнения (18) существует и принадлежит интервалу $(0, \bar{\beta})$.

Достаточность условия (22) для существования у оператора $\bar{H}$ хотя бы одного положительного собственного значения доказана. Для доказательство необходимости условия $(22)$ рассмотрим три случая: $s \leqslant 0$ и $d$ произвольное, $0<s<1$ и $d=1,2$ и, наконец, $0<s<1$ и $d \geqslant 3$. 
Случай $s \leqslant 0 u d-$ произвольное. Доказательство требуемого утверждения проведем от противного, предполагая, что (22) не выполняется: $\bar{\beta} \leqslant(1-s)^{-1} \beta_{\mathrm{c}}$. Покажем, что при этом уравнение $(23)$ не имеет положительных решений. Вначале предположим, что такие решения существуют. Так как $s \leqslant 0$, то $1-s>0$ и $G_{\lambda}^{-1}=(1-s) \bar{\beta}+s \lambda \leqslant \beta_{\mathrm{c}}+s \lambda$, что равносильно

$$
G_{\lambda}^{-1}-\beta_{\mathrm{c}} \leqslant s \lambda .
$$

Из определения (8) функции $G_{\lambda}$ следует, что $G_{\lambda}^{-1}>\beta_{\mathrm{c}}=G_{0}^{-1}$ для любого $\lambda>0$. Значит, левая часть неравенства (27) положительна при любых $\lambda>0$, а правая - отрицательна, и поэтому неравенство не выполняется. Приходим к противоречию с предположением. Доказательство необходимости условия (22) для существования у оператора $\bar{H}$ хотя бы одного положительного собственного значения в случае $s \leqslant 0$ и произвольного $d$ завершено.

Случай $0<s<1 u d=1,2$. Если оператор $\bar{H}$ обладает положительным собственным значением $\lambda$, то по лемме 3.2 это собственное значение является решением уравнения (18), которое может быть переписано в виде

$$
\bar{\beta}=(1-s)^{-1}\left(G_{\lambda}^{-1}-s \lambda\right) .
$$

При $d=1,2$ из асимптотического разложения (12) вытекает, что интеграл в правой части (8) расходится и $\beta_{\mathrm{c}}=G_{0}^{-1}=0$, так что при $0<s<1$ условие (22) примет вид $\bar{\beta}>0$. Из (28) следует, что последнее неравенство может быть переписано при $0<s<1$ в виде $G_{\lambda}^{-1}>s \lambda$. Противоположное неравенство $G_{\lambda}^{-1} \leqslant s \lambda$ не выполняется ни при одном $\lambda>0$, поскольку в размерностях $d=1,2$ в силу соотношения (26) оно принимает вид $\lambda(1+\lambda r(\lambda))^{-1} \leqslant s \lambda$, где $0<s<1$ и $0<1+\lambda r(\lambda)<1$. Доказательство необходимости условия (22) для существования у оператора $\bar{H}$ хотя бы одного положительного собственного значения в случае $0<s<1$ и $d=1,2$ завершено.

Случай $0<s<1 u d \geqslant 3$. Как и в предыдущем случае, если оператор $\bar{H}$ обладает положительным собственным значением $\lambda$, то по лемме 3.2 это собственное значение является решением уравнения (18), допускающего представление (28). Тогда условие (22) принимает вид $(1-s)^{-1}\left(G_{\lambda}^{-1}-s \lambda\right)>(1-s)^{-1} G_{0}^{-1}$, что равносильно

$$
G_{0}-G_{\lambda}>s \lambda G_{0} G_{\lambda}
$$

Из (12) при $d \geqslant 3$ и $\lambda=0$ следует, что $G_{0}<\infty$, а в силу (8) $G_{0}>G_{\lambda}$ для любого $\lambda>0$. Значит, при $0<s<1$ и $\lambda>0$ левая и правая части неравенства (29) положительны. Вначале покажем, что для любого $\lambda>0$ выполняется неравенство

$$
G_{0}-G_{\lambda} \geqslant \lambda G_{0} G_{\lambda}
$$


$\mathrm{C}$ помощью интегрирования по частям при $d \geqslant 3$ получаем

$$
\begin{aligned}
G_{\lambda} & =\int_{0}^{\infty} e^{-\lambda t} p(t) d t=\lambda \int_{0}^{\infty} e^{-\lambda t}\left(\int_{0}^{t} p(v) d v\right) d t \\
& =\lambda \int_{0}^{\infty} e^{-\lambda t}\left(G_{0}-\int_{t}^{\infty} p(v) d v\right) d t .
\end{aligned}
$$

Отсюда следует, что $G_{\lambda}=G_{0}-\lambda \int_{0}^{\infty} e^{-\lambda t}\left(\int_{t}^{\infty} p(v) d v\right) d t$ и, значит, левая часть неравенства (30) может быть представлена в виде

$$
G_{0}-G_{\lambda}=\lambda \int_{0}^{\infty} e^{-\lambda t}\left(\int_{t}^{\infty} p(v) d v\right) d t
$$

Для оценки функции $p(t)$ в правой части воспользуемся следующей леммой, доказательство которой вынесено в п. 3.3 .

Лемма 3.4. При каждом $d \in \mathbf{N}$ справедливо неравенство

$$
\left(p^{\prime}(t)\right)^{2} \leqslant p^{\prime \prime}(t) p(t)
$$

а при $d \geqslant 3$ выполняется еще и неравенство

$$
p(t) \leqslant G_{0}^{-1} \int_{t}^{\infty} p(v) d v
$$

Из этой леммы получаем, что

$$
\begin{aligned}
G_{0}-G_{\lambda} & =\lambda G_{0} \int_{0}^{\infty} G_{0}^{-1} e^{-\lambda t}\left(\int_{t}^{\infty} p(v) d v\right) d t \\
& \geqslant \lambda G_{0} \int_{0}^{\infty} e^{-\lambda t} p(t) d t=\lambda G_{0} G_{\lambda}
\end{aligned}
$$

отсюда вытекает (30). Так как по предположению $0<s<1$, то $\lambda G_{0} G_{\lambda}>$ $s \lambda G_{0} G_{\lambda}$. Таким образом, установлена справедливость неравенства (29).

Доказательство необходимости условия (22) для существования у оператора $\bar{H}$ хотя бы одного положительного собственного значения в случае $0<s<1$ и $d \geqslant 3$, а с ним и доказательство утверждения а) теоремы 3.2 , завершены.

Для доказательства утверждения б) достаточно установить, что при $\bar{\beta}>\bar{G}_{0}^{-1}$ оператор $\bar{H}$ имеет не более одного собственного значения $\lambda>$ 0 , удовлетворяющего уравнению (18). Но в силу (9) левая часть этого уравнения, функция $\bar{\beta} \bar{G}_{\lambda}$, монотонно убывает по $\lambda$, откуда и следует, что уравнение (18) имеет не более одного решения при $\lambda>0$. Это доказывает утверждение б), а с ним и теорему 3.2 . 


\section{3. Доказательства вспомогательных утверждений.}

Д ок аз а те льс т в о ле м мы 3.1. Заметим, что

$$
D^{2}=I+2\left(\sqrt{1+(1-s)^{-1} s}-1\right) \Delta_{0}+\left(\sqrt{1+(1-s)^{-1} s}-1\right)^{2} \Delta_{0}^{2} .
$$

Здесь $\Delta_{0}^{2}=\Delta_{0}$, откуда следует, что $D^{2}=I+(1-s)^{-1} s \Delta_{0}$, а тогда $\bar{H}=A+(1-s)^{-1} s \Delta_{0} A+\bar{\beta} \Delta_{0}=D^{2} A+\bar{\beta} \Delta_{0}$.

Поскольку в силу равенства $\Delta_{0}^{2}=\Delta_{0}$ спектр $\Delta_{0}$ состоит из точек 0 и 1 , а в силу условия $s<1$ леммы 3.1 справедливо неравенство $(1-s)^{-1} s>-1$, то оператор $D=I+\left(\sqrt{1+(1-s)^{-1} s}-1\right) \Delta_{0}$ обратим. Значит,

$$
D^{-1} \bar{H} D=D^{-1} D^{2} A D+\bar{\beta} D^{-1} \Delta_{0} D=D A D+\bar{\beta} D^{-1} \Delta_{0} D .
$$

Здесь $D^{-1} \Delta_{0} D=\Delta_{0}$, поскольку операторы $D$ и $D^{-1}$ коммутируют с $\Delta_{0}$. Поэтому

$$
D^{-1} \bar{H} D=D A D+\bar{\beta} \Delta_{0} .
$$

Так как операторы $\Delta_{0}$ и $A$ являются самосопряженными, то самосопряженными являются также оператор $D=I+\left(\sqrt{1+(1-s)^{-1} s}-1\right) \Delta_{0}$ и оператор $D A D$ в правой части (33). Отсюда следует самосопряженность оператора $D^{-1} \bar{H} D$. Лемма доказана.

Д о к а з а т е л ь с т в о л е м м ы 3.2. По определению, для того чтобы число $\lambda$ было собственным значением оператора $\bar{H}$, необходимо и достаточно, чтобы нашелся ненулевой элемент $g \in l^{2}\left(\mathbf{Z}^{d}\right)$, удовлетворяющий уравнению

$$
(\bar{H}-\lambda I) g=\left(\bar{A}+\bar{\beta} \Delta_{0}-\lambda I\right) g=0,
$$

которое в силу равенства $\Delta_{0} g=g(0) \delta_{0}$ можно представить в виде

$$
(\bar{A} g)(x)+\bar{\beta} g(0) \delta_{0}(x)=\lambda g(x), \quad x \in \mathbf{Z}^{d} .
$$

$\mathrm{C}$ помощью доказанного ранее равенства $\bar{A}=A+(1-s)^{-1} s \Delta_{0} A$ последнее уравнение может быть переписано как

$$
(A g)(x)+s(1-s)^{-1}\left(\sum_{x^{\prime}} a\left(x^{\prime}\right) g\left(x^{\prime}\right)\right) \delta_{0}(x)+\bar{\beta} g(0) \delta_{0}(x)=\lambda g(x), \quad x \in \mathbf{Z}^{d} .
$$

Здесь, как уже отмечалось выше, ограниченность оператора $A$ вытекает из леммы Шура [23], [20], а его самосопряженность - из симметричности матрицы $(a(x, y))_{x, y \in \mathbf{Z}^{d}}$.

В силу ограниченности оператора $A$ в $l^{2}\left(\mathbf{Z}^{d}\right)$ при каждом $g \in l^{2}\left(\mathbf{Z}^{d}\right)$ элемент $\psi=A g$ принадлежит $l^{2}\left(\mathbf{Z}^{d}\right)$, и для него определено преобразование Фурье $\widehat{\psi}(\theta)$, имеющее вид

$$
\widehat{\psi}(\theta)=\sum_{x \in \mathbf{Z}^{d}} \psi(x) e^{i(x, \theta)}=\sum_{x \in \mathbf{Z}^{d}}\left(\sum_{x^{\prime} \in \mathbf{Z}^{d}} a\left(x-x^{\prime}\right) g\left(x^{\prime}\right)\right) e^{i\left(x-x^{\prime}+x^{\prime}, \theta\right)}=\phi(\theta) \widehat{g}(\theta),
$$


где $\phi(\theta)=\sum_{x} a(x) \cos (x, \theta),(\cdot, \cdot)$ - евклидово скалярное произведение в $\mathbf{Z}^{d}$ и $\widehat{g}(\theta)$ - преобразование Фурье функции $g(x)$.

Применим к уравнению (36) преобразование Фурье:

$$
\phi(\theta) \widehat{g}(\theta)+s(1-s)^{-1} \sum_{x^{\prime}} a\left(x^{\prime}\right) g\left(x^{\prime}\right)+\bar{\beta} g(0)=\lambda \widehat{g}(\theta), \quad x \in[-\pi, \pi]^{d} .
$$

Из разрешимости уравнения (37) в $l^{2}\left([-\pi, \pi]^{d}\right)$ относительно функции $\widehat{g}(\theta)$ и из обратимости преобразования Фурье в $l^{2}$ получаем, что уравнение (35), а с ним и (34), разрешимо, если разрешимо уравнение (37).

Итак, уравнение (35) имеет ненулевое решение в $l^{2}\left(\mathbf{Z}^{d}\right)$ тогда и только тогда, когда уравнение (37) имеет ненулевое решение в $l^{2}\left([-\pi, \pi]^{d}\right)$, или, что то же самое, тогда и только тогда, когда выполнены следующие условия:

$$
\widehat{g}(\theta) \not \equiv 0, \quad \widehat{g}(\theta) \in l^{2}\left([-\pi, \pi]^{d}\right), \quad \widehat{g}(\theta)=\frac{\nu}{\lambda-\phi(\theta)},
$$

где $\nu=\bar{\beta} g(0)+s(1-s)^{-1} \sum_{x^{\prime}} a\left(x^{\prime}\right) g\left(x^{\prime}\right)$. Второе условие в (38) является, очевидно, следствием третьего при $\lambda>0$, поскольку в этом случае $\lambda-$ $\phi(\theta)>0$ на $[-\pi, \pi]^{d}$, и, значит, правая часть третьего равенства в $(38)$ есть непрерывная функция на $[-\pi, \pi]^{d}$.

Найдем явный вид функции $g(x)$, удовлетворяющей соотношениям (38). Применяя к третьему уравнению в (38) обратное преобразование Фурье, получим, что функция $g(x)$ определяется равенством

$$
g(x)=\frac{\nu}{(2 \pi)^{d}} \int_{[-\pi, \pi]^{d}} \frac{e^{-i(\theta, x)}}{\lambda-\phi(\theta)} d \theta, \quad x \in \mathbf{Z}^{d} .
$$

Заметим теперь, что, как показано в [23],

$$
\frac{1}{(2 \pi)^{d}} \int_{[-\pi, \pi]^{d}} \frac{e^{-i(\theta, y-x)}}{\lambda-\phi(\theta)} d \theta \equiv G_{\lambda}(x, y),
$$

и поэтому $g(x) \not \equiv 0$ тогда и только тогда, когда $\nu \neq 0$. Более того, положив в (39) $x=0$, получаем, что $\nu=g(0) G_{\lambda}^{-1}$, тогда $g(x)=$ $g(0) G_{\lambda}(x, 0) G_{\lambda}^{-1}$. Но в силу (10) и $(11) G_{\lambda}(x, 0) G_{\lambda}^{-1}=\bar{G}_{\lambda}(x, 0) \bar{G}_{\lambda}^{-1}$. Отсюда вытекает (19), а также тот факт, что подпространство собственных векторов оператора $\bar{H}$, отвечающих собственному значению $\lambda>0$, одномерно. Но поскольку по лемме 3.1 оператор $\bar{H}$ подобен самосопряженному, то каждое его собственное значение с одномерным пространством собственных векторов простое. Наконец, по определению $\nu=\bar{\beta} g(0)+s(1-s)^{-1} \sum_{x} a(x) g(x)$, откуда

$$
\nu=\frac{\nu}{(2 \pi)^{d}} \int_{[-\pi, \pi]^{d}} \frac{\bar{\beta}+s(1-s)^{-1} \phi(\theta)}{\lambda-\phi(\theta)} d \theta .
$$


Здесь при выполнении требования $g(x) \not \equiv 0$ справедливо неравенство $\nu \neq 0$. Поэтому, разделив левую и правую часть последнего уравнения на $\nu \neq 0$, получаем

$$
\frac{1}{(2 \pi)^{d}} \int_{[-\pi, \pi]^{d}} \frac{\bar{\beta}+s(1-s)^{-1} \phi(\theta)}{\lambda-\phi(\theta)} d \theta=1 .
$$

Теперь представим левую часть этого уравнения как

$$
\begin{array}{r}
\frac{1}{(2 \pi)^{d}} \int_{[-\pi, \pi]^{d}} \frac{\bar{\beta}-s(1-s)^{-1}(\lambda-\phi(\theta)-\lambda)}{\lambda-\phi(\theta)} d \theta \\
=\left(\bar{\beta}+s(1-s)^{-1} \lambda\right) G_{\lambda}-s(1-s)^{-1} .
\end{array}
$$

Тогда уравнение (41) принимает вид $(1-s)\left(\bar{\beta}+s(1-s)^{-1} \lambda\right) G_{\lambda}=1$, что в силу (11) равносильно (18). Лемма доказана.

Д ок аз а т е ль с т в о л е м м ы 3.3. Пусть $D$ - оператор, определенный формулой (21). Тогда по лемме 3.1 оператор $\check{H}=D^{-1} \bar{H} D$ самосопряженный. Пусть $g-$ собственный вектор оператора $\bar{H}$, отвечающий собственному значению $\lambda$. Тогда вектор $\check{g}=D^{-1} g$ будет собственным вектором, отвечающим собственному значению $\lambda$ оператора $\check{H}$, т.е. $\check{H} \check{g}=\lambda \check{g}$. Обозначим $\check{P}$ спектральный проектор оператора $\check{H}$ на одномерное подпространство, натянутое на собственный вектор $\check{g}$. В этом случае $\check{P}$ является ортопроектором [9] и имеет следующий вид:

$$
\check{P} \check{u}=\frac{\langle\check{g}, \check{u}\rangle}{\langle\check{g}, \check{g}\rangle} \check{g} .
$$

Поскольку спектральный проектор $\bar{P}$ оператора $\bar{H}$ на одномерное собственное подпространство, отвечающее простому собственному значению $\lambda>0$, связан с проектором $\check{P}$ соотношением $\bar{P}=D \check{P} D^{-1}$, то

$$
\bar{P} u=\frac{\left\langle D^{-1} g, D^{-1} u\right\rangle}{\left\langle D^{-1} g, D^{-1} g\right\rangle} g .
$$

Поэтому для вычисления явного вида оператора (42) необходимо найти оператор $D^{-1}$.

Как и в лемме 3.1 при нахождении оператора $D$, оператор $D^{-1}$ естественно искать в виде $D^{-1}=I+\varkappa \delta_{0} \delta_{0}^{T}$, исходя из уравнения $\left(I+\varkappa \delta_{0} \delta_{0}^{T}\right)\left(I+\varrho \delta_{0} \delta_{0}^{T}\right)=I$, где в силу $(21) \rho=\sqrt{1+(1-s)^{-1} s}-1$. Тогда $\varkappa$ должно быть корнем уравнения $\varkappa+\varrho+\varkappa \varrho=0$, и, значит,

$$
\varkappa=-\frac{\varrho}{1+\varrho} \text {. }
$$

Следовательно,

$$
D^{-1}=I+\varkappa \Delta_{0}, \quad \varkappa=\frac{1-\sqrt{1+(1-s)^{-1} s}}{\sqrt{1+(1-s)^{-1} s}} .
$$


Подставив полученное выражение для $D^{-1}$ в (42), получаем

$$
\bar{P} u=\frac{\left\langle g+\varkappa \Delta_{0} g, u+\varkappa \Delta_{0} u\right\rangle}{\left\langle g+\varkappa \Delta_{0} g, g+\varkappa \Delta_{0} g\right\rangle} g,
$$

откуда

$$
\bar{P} u=\frac{\langle g, u\rangle-s\left\langle\delta_{0}, g\right\rangle\left\langle\delta_{0}, u\right\rangle}{\langle g, g\rangle-s\left\langle\delta_{0}, g\right\rangle^{2}} g .
$$

Доказательство леммы 3.3 завершено.

Д о к а з а т е л ь с т в о л е м м ы 3.4. В [14], [23], [24] доказано, что

$$
\begin{aligned}
p(t) & =\frac{1}{(2 \pi)^{d}} \int_{[-\pi, \pi]^{d}} e^{\phi(\theta) t} d \theta, \\
p^{\prime}(t) & =\frac{1}{(2 \pi)^{d}} \int_{[-\pi, \pi]^{d}} \phi(\theta) e^{\phi(\theta) t} d \theta, \\
p^{\prime \prime}(t) & =\frac{1}{(2 \pi)^{d}} \int_{[-\pi, \pi]^{d}} \phi^{2}(\theta) e^{\phi(\theta) t} d \theta,
\end{aligned}
$$

где функция $\phi(\theta)=\sum_{x} a(x, 0) e^{i(x, \theta)}$ дважды непрерывно дифференцируема и принимает вещественные значения при $\theta \in[-\pi, \pi]^{d}$. Из неравенства Коши-Буняковского в интегральной форме вытекает, что

$$
\int_{[-\pi, \pi]^{d}} e^{\phi(\theta) t} d \theta \int_{[-\pi, \pi]^{d}} \phi^{2}(\theta) e^{\phi(\theta) t} d \theta \geqslant\left(\int_{[-\pi, \pi]^{d}} e^{\phi(\theta) t / 2} \phi(\theta) e^{\phi(\theta) t / 2} d \theta\right)^{2}
$$

и, следовательно, неравенство (31) выполнено.

Для доказательства неравенства (32) заметим, что в силу (8) $G_{0}=$ $\int_{0}^{\infty} p(u) d u$, и поэтому (32) равносильно неравенству $p(t) \int_{0}^{\infty} p(u) d u \leqslant$ $\int_{t}^{\infty} p(u) d u$, которое с учетом начального условия $p(0)=1$ может быть представлено в виде

$$
\int_{0}^{\infty} \frac{p(u)}{p(0)} d u \leqslant \int_{t}^{\infty} \frac{p(u)}{p(t)} d u
$$

Проведя замену переменных $u=t+\tau$ в правом интеграле, получим

$$
\int_{0}^{\infty} \frac{p(u)}{p(0)} d u \leqslant \int_{0}^{\infty} \frac{p(t+\tau)}{p(t)} d \tau
$$

Чтобы доказать последнее неравенство, достаточно установить, что подынтегральная функция $p(t+\tau) / p(t)$ монотонно не убывает по $t$ при каждом $\tau$, т.е. что при $v \geqslant z$ справедливо неравенство $p(v+\tau) / p(v) \geqslant$ $p(z+\tau) / p(z)$, равносильное соотношению

$$
\frac{d}{d t}\left(\frac{p(t+\tau)}{p(t)}\right) \geqslant 0
$$


Очевидно, это соотношение выполняется тогда и только тогда, когда

$$
\frac{p^{\prime}(t+\tau) p(t)-p^{\prime}(t) p(t+\tau)}{p^{2}(t)} \geqslant 0
$$

или $p^{\prime}(t+\tau) / p(t+\tau) \geqslant p^{\prime}(t) / p(t)$. Последнее неравенство может быть переписано в виде соотношения $(\ln p(t+\tau))^{\prime} \geqslant(\ln p(t))^{\prime}$, которое выполняется тогда и только тогда, когда функция $(\ln p(t))^{\prime}$ не убывает, что равносильно $(\ln p(t))^{\prime \prime} \geqslant 0$ или

$$
(\ln p(t))^{\prime \prime}=\frac{p^{\prime \prime}(t) p(t)-\left(p^{\prime}(t)\right)^{2}}{p^{2}(t)} \geqslant 0 \quad \Longleftrightarrow \quad p^{\prime \prime}(t) p(t) \geqslant\left(p^{\prime}(t)\right)^{2} .
$$

Справедливость неравенства в правой части последнего соотношения уже установлена выше, и значит, доказательство неравенства (32) завершено. Лемма доказана.

4. Предельная теорема для ВСБ в модели II. По теоремам 3.1 и 3.2 при $\bar{\beta}>\bar{G}_{0}^{-1}$ спектр оператора $\bar{H}$, действующего в $l^{2}\left(\mathbf{Z}^{d}\right)$, pacпадается на часть, принадлежащую неположительной действительной полуоси, и изолированное положительное собственное значение, которое является решением уравнения (18). Далее собственное значение оператора $\bar{H}$ будем обозначать $\bar{\lambda}$. Положим $\bar{\beta}_{c}=\bar{G}_{0}^{-1}$.

Лемма 4.1. Пусть $V$ - ограниченный оператор в банаховом пространстве $\mathfrak{B}$, спектр которого распадается на два непересекающихся множества: изолированное простое собственное значение $\lambda>0$ u часть, принадлежащую полупростанству $\{z \in \mathbf{C}: \operatorname{Re} z \leqslant \sigma<\lambda\}$. Тогда для решения $и(t)$ задачи Коши

$$
\frac{d u}{d t}=V u, \quad u(0)=u_{0},
$$

выполняется соотношение $\lim _{t \rightarrow \infty} e^{-\lambda t} u(t)=P u_{0}$, где $P-$ спектральньй проектор оператора $V$ на собственное подпространство, отвечаюшее собственному значению $\lambda$.

Эта лемма является известным фактом теории дифференциальных уравнений в банаховых пространствах [9]. В [23] аналогичное утверждение доказано для случая, когда $V$ является самосопряженным оператором в гильбертовом пространстве.

4.1. Асимптотика моментов локальных численностей частиц. Начнем изучение поведения численностей частиц с локальных численностей.

Теорема 4.1. Пусть $\bar{\beta}>\bar{\beta}_{\mathrm{c}}$. Тогда при всех $n \in \mathbf{N} u t \rightarrow \infty$ справедливь соотношения

$$
m_{n}(t ; x, y) \sim C_{n}(x, y) e^{n \bar{\lambda} t}
$$


где

$$
\begin{aligned}
C_{1}(x, 0) & =\frac{(1-s) \bar{G}_{\bar{\lambda}}(x, 0) \bar{G}_{\bar{\lambda}}}{\left\|\bar{G}_{\bar{\lambda}}(\cdot, 0)\right\|^{2}-s \bar{G}_{\bar{\lambda}}^{2}}, \\
C_{1}(x, y) & =\frac{\bar{G}_{\bar{\lambda}}(x, 0) \bar{G}_{\bar{\lambda}}(y, 0)}{\left\|\bar{G}_{\bar{\lambda}}(\cdot, 0)\right\|^{2}-s \bar{G}_{\bar{\lambda}}^{2}} \quad \text { npu } \quad y \neq 0,
\end{aligned}
$$

$\|\cdot\|$ - норма в $l^{2}\left(\mathbf{Z}^{d}\right)$, а при $n \geqslant 2$ функции $C_{n}(x, y)$ определяются рекуррентно:

$$
C_{n}(x, y)=g_{n}\left(C_{1}(0, y), \ldots, C_{n-1}(0, y)\right) \frac{\bar{G}_{n \bar{\lambda}}(x, 0)}{1-\bar{\beta} \bar{G}_{n \bar{\lambda}}},
$$

где функиии $g_{n}$ определень равенствами (15).

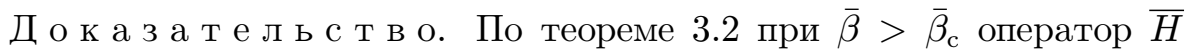
обладает простым собственным значением $\bar{\lambda}$, являющимся решением уравнения (18). При этом собственный вектор $g$ оператора $\bar{H}$, отвечающий собственному значению $\bar{\lambda}$, определяется равенством (19). Докажем вначале соотношение (43) для $n=1$. Применив лемму 4.1 к случаю, когда $V=\bar{H}, \mathfrak{B}=l^{2}\left(\mathbf{Z}^{d}\right)$, получаем, что

$$
\lim _{t \rightarrow \infty} m_{1}(t ; \cdot, y) e^{-\bar{\lambda} t}=\bar{P} \delta_{y},
$$

где $\bar{\lambda}$ является решением уравнения (18). Здесь в силу утверждения в) теоремы 3.1

$$
\begin{aligned}
& \bar{P} \delta_{0}=\frac{\left\langle g, \delta_{0}\right\rangle-s\left\langle\delta_{0}, g\right\rangle\left\langle\delta_{0}, \delta_{0}\right\rangle}{\langle g, g\rangle-s\left\langle\delta_{0}, g\right\rangle^{2}} g=\frac{(1-s) \bar{G}_{\bar{\lambda}}(\cdot, 0) \bar{G}_{\bar{\lambda}}}{\left\|\bar{G}_{\bar{\lambda}}(\cdot, 0)\right\|^{2}-s \bar{G}_{\bar{\lambda}}^{2}}, \\
& \bar{P} \delta_{y}=\frac{\left\langle g, \delta_{y}\right\rangle-s\left\langle\delta_{0}, g\right\rangle\left\langle\delta_{0}, \delta_{y}\right\rangle}{\langle g, g\rangle-s\left\langle\delta_{0}, g\right\rangle^{2}} g=\frac{\bar{G}_{\bar{\lambda}}(\cdot, 0) \bar{G}_{\bar{\lambda}}(y, 0)}{\left\|\bar{G}_{\bar{\lambda}}(\cdot, 0)\right\|^{2}-s \bar{G}_{\bar{\lambda}}^{2}} \quad \text { при } y \neq 0 .
\end{aligned}
$$

Поэтому в координатной форме соотношение (46) представляется в виде

$$
\begin{aligned}
\lim _{t \rightarrow \infty} m_{1}(t ; x, 0) e^{-\bar{\lambda} t} & =\frac{(1-s) \bar{G}_{\bar{\lambda}}(x, 0) \bar{G}_{\bar{\lambda}}}{\left\|\bar{G}_{\bar{\lambda}}(\cdot, 0)\right\|^{2}-s \bar{G}_{\bar{\lambda}}^{2}}, \\
\lim _{t \rightarrow \infty} m_{1}(t ; x, y) e^{-\bar{\lambda} t} & =\frac{\bar{G}_{\bar{\lambda}}(x, 0) \bar{G}_{\bar{\lambda}}(y, 0)}{\left\|\bar{G}_{\bar{\lambda}}(\cdot, 0)\right\|^{2}-s \bar{G}_{\bar{\lambda}}^{2}} \quad \text { при } y \neq 0 .
\end{aligned}
$$

Для доказательство соотношений (45) введем функции $\nu_{n}(t ; x, y)=$ $m_{n}(t ; x, y) e^{-n \bar{\lambda} t}, n \geqslant 1$. Тогда из (13) и (14) получаем уравнения для $\nu_{n}$ :

$$
\begin{aligned}
\partial_{t} \nu_{1} & =\bar{H} \nu_{1}-\bar{\lambda} \nu_{1} \\
\partial_{t} \nu_{n} & =\bar{H} \nu_{n}-n \bar{\lambda} \nu_{n}+\delta_{0}(x) g_{n}\left(\nu_{1}, \ldots, \nu_{n-1}\right), \quad n \geqslant 2,
\end{aligned}
$$

с начальными условиями $\nu_{n}(0 ; \cdot, y)=\delta_{y}(\cdot), n \geqslant 1$. 
Поскольку $\bar{\lambda}>0$ является изолированным собственным значением оператора $\bar{H}$, то при $n \geqslant 2$ спектр оператора $\widetilde{H}_{n}=\bar{H}-n \bar{\lambda} I$ содержит отрицательное изолированное собственное значение $\sigma=-(n-1) \bar{\lambda}$ и часть спектра, расположенную левее точки $\sigma$. Воспользуемся следующим очевидным утверждением теории дифференциальных уравнений: если спектр ограниченного оператора $\breve{H}$ в гильбертовом пространстве принадлежит $(-\infty, \sigma], \sigma<0$, и $f(t) \rightarrow f_{*}$ при $t \rightarrow \infty$, то для решения уравнения

$$
\frac{d \nu}{d t}=\breve{H} \nu+f(t)
$$

выполняется соотношение $\nu(t) \rightarrow \breve{H}^{-1} f_{*}$. Трактуя теперь каждое из уравнений $(47),(48)$ как дифференциальное уравнение в банаховом пространстве $l^{2}\left(\mathbf{Z}^{d}\right)$, в силу сформулированного утверждения и асимптотического поведения $m_{1}$ при $t \rightarrow \infty$ получаем, что

$$
\begin{aligned}
C_{1}(x, 0) & =\lim _{t \rightarrow \infty} \nu_{1}(t ; x, 0)=\frac{(1-s) \bar{G}_{\bar{\lambda}}(x, 0) \bar{G}_{\bar{\lambda}}}{\left\|\bar{G}_{\bar{\lambda}}(x, 0)\right\|^{2}-s \bar{G}_{\bar{\lambda}}^{2}} \\
C_{n}(x, 0) & =\lim _{t \rightarrow \infty} \nu_{n}(t ; x, 0) \\
& =-g_{n}\left(C_{1}(0,0), \ldots, C_{n-1}(0,0)\right) \widetilde{H}_{n}^{-1} \delta_{0}(x), \quad n \geqslant 2 .
\end{aligned}
$$

Нахождение элемента $\widetilde{H}_{n}^{-1} \delta_{0}$ в последнем равенстве в силу соотношения $h=\widetilde{H}_{n}^{-1} \delta_{0}=(\bar{H}-n \bar{\lambda} I)^{-1} \delta_{0}$ равносильно решению уравнения $(\bar{H}-$ $n \bar{\lambda} I) h=\delta_{0}$, которое в силу равенства $\bar{H}=\left(I+(1-s)^{-1} s \Delta_{0}\right) A+\bar{\beta} \Delta_{0}$ может быть переписано в виде

$$
\left(A+(1-s)^{-1} s \Delta_{0} A+\bar{\beta} \Delta_{0}-n \bar{\lambda} I\right) h=\delta_{0} .
$$

А так как $\Delta_{0} h=h(0) \delta_{0}$, то (49) допускает следующее «координатное» представление:

$$
(A h)(x)+(1-s)^{-1} s\left(\sum_{x^{\prime}} a\left(x^{\prime}\right) h\left(x^{\prime}\right)\right) \delta_{0}(x)+\bar{\beta} h(0) \delta_{0}(x)-n \bar{\lambda} h(x)=\delta_{0}(x) .
$$

Применяя к этому уравнению преобразование Фурье и используя обозначения, введенные при доказательстве леммы 3.2 , получаем

$\phi(\theta) \hat{h}(\theta)+s(1-s)^{-1} \sum_{x^{\prime}} a\left(x^{\prime}\right) h\left(x^{\prime}\right)+\bar{\beta} h(0)-n \bar{\lambda} \hat{h}(\theta)=1, \quad x \in[-\pi, \pi]^{d}$,

где $\hat{h}(\theta)$ - преобразование Фурье функции $h(x)$. Положив здесь

$$
\rho=\bar{\beta} h(0)+s(1-s)^{-1} \sum_{x^{\prime}} a\left(x^{\prime}\right) h\left(x^{\prime}\right)-1,
$$

получаем

$$
\hat{h}(\theta)=\rho(n \bar{\lambda}-\phi(\theta))^{-1} .
$$


Наконец, применяя обратное преобразование Фурье к (51), находим решение уравнения (49):

$$
h(x)=\frac{\rho}{(2 \pi)^{d}} \int_{[-\pi, \pi]^{d}} \frac{e^{-i(\theta, x)}}{n \bar{\lambda}-\phi(\theta)} d \theta .
$$

Осталось вычислить значение $\rho$. Подставив в $(50)$ значения $h(0)$ и $h(x)$ из (52), получаем

$$
\rho=\frac{\rho}{(2 \pi)^{d}} \int_{[-\pi, \pi]^{d}} \frac{\bar{\beta}+s(1-s)^{-1} \phi(\theta)}{n \bar{\lambda}-\phi(\theta)} d \theta-1 .
$$

Здесь в силу (40)

$$
\frac{1}{(2 \pi)^{d}} \int_{[-\pi, \pi]^{d}} \frac{e^{-i(\theta, x)}}{n \bar{\lambda}-\phi(\theta)} d \theta=G_{n \bar{\lambda}}(x, 0),
$$

и поэтому

$$
\rho=\frac{1-s}{(\bar{\beta}(1-s)+s n \bar{\lambda}) G_{n \bar{\lambda}}-1} .
$$

$\mathrm{C}$ помощью последнего равенства из (52) получаем

$$
h(x)=\frac{(1-s) G_{n \bar{\lambda}}(x, 0)}{(\bar{\beta}(1-s)+s n \bar{\lambda}) G_{n \bar{\lambda}}-1},
$$

где в силу (10)

$$
G_{n \bar{\lambda}}(x, 0)=\frac{\bar{G}_{n \bar{\lambda}}(x, 0)}{1+s\left(n \bar{\lambda} \bar{G}_{n \bar{\lambda}}-1\right)} .
$$

Теперь выражение для $h(x)$ может быть переписано в терминах модели II:

$$
h(x)=-\frac{\bar{G}_{n \bar{\lambda}}(x, 0)}{1-\bar{\beta} \bar{G}_{n \bar{\lambda}}} .
$$

Следовательно, при $n \geqslant 2$

$$
C_{n}(x, 0)=g_{n}\left(C_{1}(0,0), \ldots, C_{n-1}(0,0)\right) \frac{\bar{G}_{n \bar{\lambda}}(x, 0)}{1-\bar{\beta} \bar{G}_{n \bar{\lambda}}} .
$$

Таким образом, найдена асимптотика при $t \rightarrow \infty$ моментов $m_{n}(t ; x, 0)$ локальной численности частиц $\bar{\mu}_{t}(0)$ на $\mathbf{Z}^{d}$.

Применяя теперь метод вывода формул для $C_{n}(x, 0)$, нетрудно получить выражения для $C_{n}(x, y)$ и показать, что поведение моментов $m_{n}(t ; x, y)$ локальной численности частиц $\bar{\mu}_{t}(y)$ при $y \neq 0$ определяется следуюшими соотношениями:

$$
\begin{aligned}
C_{1}(x, y) & =\lim _{t \rightarrow \infty} \nu_{1}(t ; x, y)=\frac{\bar{G}_{\bar{\lambda}}(x, 0) \bar{G}_{\bar{\lambda}}(y, 0)}{\left\|\bar{G}_{\bar{\lambda}}(\cdot, 0)\right\|^{2}-s \bar{G}_{\bar{\lambda}}^{2}} \\
C_{n}(x, y) & =\lim _{t \rightarrow \infty} \nu_{n}(t ; x, y) \\
& =-g_{n}\left(C_{1}(0, y), \ldots, C_{n-1}(0, y)\right) \widetilde{H}_{n}^{-1} \delta_{0}(x), \quad n \geqslant 2 .
\end{aligned}
$$


Здесь величины $\widetilde{H}_{n}^{-1} \delta_{0}(x)$ уже вычислены, и поэтому задача описания асимптотического поведения $\nu_{n}(t ; x, y)=m_{n}(t ; x, y) e^{-n \bar{\lambda} t}$ при $y \neq 0$ полностью решена. Теорема доказана.

4.2. Асимптотика моментов общих численностей частиц. Отметим следующий аналог теоремы 4.1 для общих численностей частиц.

Теорема 4.2. Пусть $\bar{\beta}>\bar{\beta}_{c}$. Тогда при всех $n \in \mathbf{N} u t \rightarrow \infty$ справедливы соотношения

$$
m_{n}(t ; x) \sim C_{n}(x) e^{n \bar{\lambda} t}
$$

где

$$
C_{1}(x)=\frac{(1-s) \bar{G}_{\bar{\lambda}}(x, 0)}{\bar{\lambda}\left(\left\|\bar{G}_{\bar{\lambda}}(\cdot, 0)\right\|^{2}-s \bar{G}_{\bar{\lambda}}^{2}\right)},
$$

a при $n \geqslant 2$ функции $C_{n}(x)$ определяются рекуррентно:

$$
C_{n}(x)=g_{n}\left(C_{1}(0), \ldots, C_{n-1}(0)\right) \frac{\bar{G}_{n \bar{\lambda}}(x, 0)}{1-\bar{\beta} \bar{G}_{n \bar{\lambda}}} .
$$

Для обоснования этой теоремы заметим, что функция $m_{1}(t ; x)=$ $\sum_{y \in \mathbf{Z}^{d}} m_{1}(t ; x, y)$ удовлетворяет уравнению (14). Данное уравнение должно рассматриваться в пространстве $l^{\infty}\left(\mathbf{Z}^{d}\right)$ и его анализ опирается на следующее обобщение теорем 3.1 и 3.2 для общего случая пространств $l^{p}\left(\mathbf{Z}^{d}\right), 1 \leqslant p \leqslant \infty$.

Теорема 4.3. Пусть $s<1$, тогда при каждом $1 \leqslant p \leqslant \infty$ оператор $\bar{H}$ является линейным ограниченныл оператором в $l^{p}\left(\mathbf{Z}^{d}\right)$ и него справедливы все утверждения теорем 3.1 и 3.2.

Доказательство теоремы 4.3 проводится по той же схеме, что и доказательство теоремы 3.1 , и поэтому не приводится.

Далее, из теоремы 4.3 в силу леммы 4.1 вытекает, что выполнено соотношение $\lim _{t \rightarrow \infty} m_{1}(t ; \cdot) e^{-\bar{\lambda} t}=\bar{P} u_{0}$, где $u_{0}-$ функция в $l^{\infty}\left(\mathbf{Z}^{d}\right)$, принимающая значения 1 в каждой точке решетки $\mathbf{Z}^{d}$. Тогда

$$
\bar{P} u_{0}=\frac{(1-s) \bar{G}_{\bar{\lambda}}(\cdot, 0)}{\bar{\lambda}\left(\left\|\bar{G}_{\bar{\lambda}}(\cdot, 0)\right\|^{2}-s \bar{G}_{\bar{\lambda}}^{2}\right)} .
$$

Запишем это соотношение в координатной форме:

$$
C_{1}(x)=\lim _{t \rightarrow \infty} m_{1}(t ; x) e^{-\bar{\lambda} t}=\frac{(1-s) \bar{G}_{\bar{\lambda}}(x, 0)}{\bar{\lambda}\left(\left\|\bar{G}_{\bar{\lambda}}(\cdot, 0)\right\|^{2}-s \bar{G}_{\bar{\lambda}}^{2}\right)} .
$$

Осталось заметить, что для вычисления асимптотики старших моментов $m_{n}(t ; x)$ при $n \geqslant 2$ теперь достаточно на основе дифференциальных уравнений (13) выписать интегральные уравнения для моментов $m_{n}(t ; x)$ и затем их индуктивно проинтегрировать. 
4.3. Предельная теорема для модели II. Начнем с доказательства леммы, устанавливающей связь между коэффициентами $C_{n}(x, y)$ и $C_{n}(x)$.

Лемма 4.2. Пусть $\bar{\beta}>\bar{\beta}_{\mathrm{c}}$. Тогда при каждом $n \in \mathbf{N}$ функиии $C_{n}(x, y)$ и $C_{n}(x)$ связаныл соотношениями

$$
C_{n}(x, y)=\psi_{\bar{\lambda}}^{n}(y) C_{n}(x)
$$

әде

$$
\psi_{\bar{\lambda}}(y)= \begin{cases}\bar{\lambda} \bar{G}_{\bar{\lambda}} & \text { npu } y=0 \\ \bar{\lambda}(1-s)^{-1} \bar{G}_{\bar{\lambda}}(0, y) & \text { npu } y \neq 0\end{cases}
$$

Д о к а з а т е л ь с т в о. Проведем доказательство по индукции. В силу (44) соотношение (54) выполняется при $n=1$. Зафиксируем произвольное $k \geqslant 2$ и предположим, что (54) верно при всех $n \leqslant k$.

Как следует из равенств (45), (53), для доказательства соотношения (54) при $n=k+1$ достаточно показать, что

$$
g_{k+1}\left(C_{1}(0, y), \ldots, C_{k}(0, y)\right)=\psi_{0}^{k+1}(y) g_{k+1}\left(C_{1}(0), \ldots, C_{k}(0)\right) .
$$

Из определения (15) функций $g_{n}$, а также предположения индукции получаем:

$$
\begin{aligned}
g_{k+1} & \left(C_{1}(0, y), \ldots, C_{k}(0, y)\right) \\
& =\sum_{r=2}^{k+1} \frac{\beta^{(r)}}{r !} \sum_{\substack{i_{1}, \ldots, i_{r}>0 \\
i_{1}+\cdots+i_{r}=k+1}} \frac{(k+1) !}{i_{1} ! \cdots i_{r} !} \psi_{0}^{i_{1}+\cdots+i_{r}}(y) C_{i_{1}}(0) \cdots C_{i_{r}}(0) \\
& =\psi_{0}^{k+1}(y) g_{k+1}\left(C_{1}(0), \ldots, C_{k}(0)\right)
\end{aligned}
$$

Шаг индукции завершен. Лемма доказана.

Теорема 4.4. Если $\bar{\beta}>\bar{\beta}_{\mathrm{c}}$, то в смилле сходимости моментов

$$
\lim _{t \rightarrow \infty} \bar{\mu}_{t}(y) e^{-\bar{\lambda} t}=\xi \psi_{\bar{\lambda}}(y), \quad \lim _{t \rightarrow \infty} \bar{\mu}_{t} e^{-\bar{\lambda} t}=\xi
$$

где функиия $\psi_{\bar{\lambda}}(y)$ определена равенством (54), а $\xi$ - невьрожденная случайная величина такая, что $\mathbf{E}_{x} \xi^{n}=C_{n}(x), n \in \mathbf{N}$ ( а величины $C_{n}(x)$ определеньл в (43)). Более того, если $\bar{\beta}_{r}=O\left(r ! r^{r-1}\right)$, то моменты $\mathbf{E}_{x} \xi^{n}$ однозначно определяют распределение $\xi$ и, таким образом, соотношения (55) справедливь также и в смьсле сходимости по распределению.

Д о к а з а т е ль с т в о. Определим при $\beta>\beta_{\text {с функции }}$

$$
\begin{aligned}
m(n, x, y) & =\lim _{t \rightarrow \infty} \frac{\mathbf{E}_{x} \mu_{t}^{n}(y)}{m_{1}^{n}(t ; x, y)}=\lim _{t \rightarrow \infty} \frac{m_{n}(t ; x, y)}{m_{1}^{n}(t ; x, y)}=\frac{C_{n}(x, y)}{C_{1}^{n}(x, y)}, \\
m(n, x) & =\lim _{t \rightarrow \infty} \frac{\mathbf{E}_{x} \mu_{t}^{n}}{m_{1}^{n}(t ; x)}=\lim _{t \rightarrow \infty} \frac{m_{n}(t ; x)}{m_{1}^{n}(t ; x)}=\frac{C_{n}(x)}{C_{1}^{n}(x)}
\end{aligned}
$$


Из леммы 4.2 вытекает, что

$$
m(n, x)=m(n, x, y)=\frac{C_{n}(x)}{C_{1}^{n}(x)}=\frac{C_{n}(x, y)}{C_{1}^{n}(x, y)} .
$$

Как известно (см. обсуждение «проблемы сходимости моментов» в [12]), если при всех $n \in \mathbf{N}$ величины $\mathbf{E}_{x} \mu_{t}^{n} / m_{1}^{n}(t ; x)$ и $\mathbf{E}_{x} \mu_{t}^{n}(y) / m_{1}^{n}(t ; x, y)$ поточечно сходятся при $t \rightarrow \infty$ к некоторым величинам $m(n, x)$, где $m(n, x)$ конечны (при каждых фиксированных $n$ и $x$ ), то $m(n, x)$ являются моментами некоторой случайной величины $\xi$ (т.е. проблема моментов разрешима). Докажем, что моменты $m(n, x)$ однозначно определяют распределение случайной величины $\xi$. Как показано в [17], [19], если $m(n, x)$ - моменты распределения, сосредоточенного на $[0, \infty)$, то для того, чтобы распределение однозначно определялось своими моментами, достаточно выполнение следующего условия (критерий Карлемана), ограничивающего скорость роста $m(n, x)$ :

$$
\sum_{n=1}^{\infty} m(n, x)^{-1 /(2 n)}=\infty
$$

С помощью (53) по индукции можно показать, в точности так же, как это сделано для первой модели [4], что $C_{n}(x) \leqslant \gamma^{n} n ! n^{n}$, где $\gamma-$ некоторая константа. Тогда выполняется условие Карлемана $\sum_{n} C_{n}^{-1 /(2 n)}=$ $\infty$, и проблема моментов Стилтьеса (на полуоси) для последовательности $\left\{C_{n}\right\}$ однозначно разрешима [17]. Теорема доказана.

Очевидно, если в теореме 4.4 число потомков частицы ограничено, то соотношения (55) всегда будут выполнены в смысле сходимости по распределению.

5. Заключительные замечания. Отметим, что ключевым моментом в исследовании асимптотик численностей частиц как в модели I в [23], так и в модели II в настоящей работе явились теоремы 3.1 и 3.2 , в которых исследованы спектральные свойства возмущенного генератора случайного блуждания $\bar{H}$ при «одноточечном» источнике ветвления, в частности установлены необходимые и достаточные условия существования «старшего» собственного значения. По-видимому, основные утверждения теорем 3.1 и 3.2 (с естественными усложнениями в формулировках) справедливы и для «многоточечных» источников ветвления (с конечным числом источников) в рассматриваемых моделях. В этом случае применение «многоточечного» варианта теорем 3.1 и 3.2 позволило бы исследовать асимптотики численностей частиц в моделях I и II с множественными точечными источниками ветвления. Таким образом, может быть предложена следующая схема исследования для нескольких источников ветвления. 
Пусть удалось установить, что спектр генератора случайного блуждания расположен на вещественной полуоси и при компактном возмущении генератора случайного блуждания, действующего в $l^{2}\left(\mathbf{Z}^{d}\right)$, появляется конечное число изолированных положительных собственных значений, старшим из которых является $\lambda$. Тогда, как и в одноточечных моделях, рассмотренных выше, можно показать, что эти условия обеспечивают экспоненциальный рост старших моментов как локальных, так и общих численностей частиц.

Пусть, кроме того, выполнены соотношения (56) при каждом $n \in \mathbf{N}$, $x \in \mathbf{Z}^{d}$ и $y \in \mathbf{Z}^{d}$. Тогда в смысле сходимости моментов для нескольких источников будут справедливы предельные соотношения типа (55), которые обеспечивают экспоненциальный рост как численности частиц в произвольном узле, так и общего числа частиц на решетке с параметром $\lambda$. Далее критерий Карлемана (57) позволит проверить, будут ли моменты однозначно определять распределение $\xi$. В случае выполнения условия (57) соотношения (55) окажутся справедливы также и в смысле сходимости по распределению.

Автор выражает благодарность Л. Г. Афанасьевой, В. А. Ватутину и А.Н. Ширяеву за полезные замечания.

\section{СПИСОК ЛИТЕРАТУРЫ}

1. Albeverio S., Bogachev L. V., Yarovaya E. B. Asymptotics of branching symmetric random walk on the lattice with a single source. - C. R. Acad. Sci. Paris, 1998, v. 326, № 9, p. 975-980.

2. Albeverio S., Bogachev L. V. Branching random walk in a catalytic medium. I. Basic equations. - Positivity, 2000, v. 4, p. 41-100.

3. Богачев Л. В., Яровая Е. Б. Моментный анализ ветвящегося случайного блуждания на решетке с одним источником. - Докл. РАН, 1998, т. 363, № 4, с. 439-442.

4. Богачев Л.В., Яровая Е. Б. Предельная теорема для надкритического ветвящегося случайного блуждания на $\mathbf{Z}^{d}$ с одним источником. - Успехи матем. наук, 1998 , в. 53 , № 5 , с. $229-230$.

5. Ватутин B. A., Топчий B. A. Предельная теорема для критических каталитических ветвящихся случайных блужданий. - Теория вероятн. и ее примен., 2004, B. 49 , № 3, c. 461-484.

6. Vatutin V.A., Topchii V.A., Yarovaya E. B. Catalytic branching random walk and queueing systems with random number of independent servers. - Teor. Imovīr. Mat. Stat., 2003, v. 69, p. 158-172.

7. Gärtner J., Molchanov S. A. Parabolic problems for the Anderson model. I: Intermittency and related topics. - Comm. Math. Phys., 1990, v. 132, № 3, p. 613-655.

8. Гихман И. И., Скороход А. В. Теория случайных процессов. Т. 2. М.: Наука, 1973, $639 \mathrm{c.}$

9. Далечкий Ю. Л., Крейн М. Г. Устойчивость решений дифференциальных уравнений в банаховом пространстве. М.: Наука, 1970, 534 с.

10. Захарьева E. В. О вероятностях выживания частиц на $\mathbf{Z}^{2}$ в одной из моделей критического ветвящегося случайного блуждания. - Труды Колмогоровских чтений, 2008, с. 218-229.

11. Като T. Теория возмущений линейных операторов. М.: Мир, 1972, 740 с. 
12. Лоэв М. Теория вероятностей. М.: ИЛ, 1962, 719 с.

13. Molchanov S. Lectures on random media. - Lecture Notes in Math., 1994, v. 1581, p. $242-411$.

14. Topchii V.A., Vatutin V. A. Individuals at the origin in the critical catalytic branching random walk. - Discrete Random Walks (Paris, 2003). Nancy: Assoc. Discrete Math. Theoret. Comput. Sci., 2003, p. 325-332.

15. Севастьянов Б. А. Ветвящиеся процессы. М.: Наука, 1971, 436 с.

16. Севастьянов Б. А. Минимальные точки надкритического ветвящегося блуждания на решетке $\mathbf{N}_{0}^{r}$ и многотипные ветвящиеся процессы Гальтона-Ватсона. Дискрет. матем., 2000, т. 12, № 1, с. 3-6.

17. Shohat J.A., Tamarkin J.D. The Problem of Moments. New York: Amer. Math. Soc., 1943,140 p.

18. Феллер B. Введение в теорию вероятностей и ее приложения. Т. 2. М.: Мир, 1984, $752 \mathrm{c}$.

19. Ширяев А.Н. Вероятность. В 2-х кн. М.: МЦНМО, 2004, 928 с.

20. Шубин М.A. Псевдоразностные операторы и их функция Грина. - Изв. АН CCCP, 1985 , т. 49 , № 3, с. $652-671$.

21. Яровая Е.Б. Применение спектральных методов в изучении ветвящихся процессов с диффузией в некомпактном фазовом пространстве. - Теорет. матем. физ., 1991, т. 88 , № 1, с. 25-30.

22. Яровая Е. Б. Предельная теорема для критического ветвящегося случайного блуждания на $\mathbf{Z}^{d}$ с одним источником. - Успехи матем. наук, 2005, т. 60, № 1, c. $175-176$.

23. Яровая Е. Б. Ветвящиеся случайные блуждания в неоднородной среде. М.: ЦПИ при мехмате Моск. ун-та, 2007, 104 с.

24. Яровая Е.Б. Критические ветвящиеся случайные блуждания по решеткам низких размерностей. - Дискрет. матем., 2009, т. 21, № 1, с. 117-138.

25. Yarovaya E. Survival analysis in nonhomogeneous branching random walks. - Sixth Conference «Mathematical Methods in Reliability». Theory. Methods. Applications (Moscow, 2009), 2009, p. 38-41.

26. Яровая Е.Б. Модели ветвящихся блужданий и их применение в теории надежности. - Автоматика и телемеханика, 2010, № 7, с. 29-46.

27. Яровая Е.Б. Об исследовании ветвящихся случайных блужданий по многомерным решеткам. - Современные проблемы математики и механики, т. 4, сер. «Теория вероятностей и математическая статистика». М.: Изд-во Моск. ун-та, 2009 , c. $119-136$.

Поступила в редакцию

29.XII.2009

Исправленный вариант

06.X.2010 